\title{
La discapacidad desde los enfoques de derechos y capacidades y su relación con la salud pública
}

\author{
Disability under the Approaches of Rights and Capabilities and How it Relates to the Public Health
}

A defciência desde os enfoques de direitos e capacidades e sua relação com a saúde pública

\author{
Natalia Eugenia Gómez Rúa ${ }^{\text {a }}$ \\ Universidad CES, Colombia \\ ngomez@ces.edu.co \\ ORCID: http://orcid.org/0000-0002-7506-8443 \\ Diego Alveiro Restrepo Ochoa \\ Universidad CES, Colombia \\ ORCID: http://orcid.org/0000-0002-4469-6260 \\ Jaime Gañan Echavarría \\ Universidad de Antioquia, Colombia \\ ORCID: http://orcid.org/0000-0002-6873-8744 \\ Doris Cardona Arango \\ Universidad CES, Colombia \\ ORCID: http://orcid.org/0000-0003-4338-588X
}

DOI: https://doi.org/10.11144/Javeriana.rgps17-35.dedc

Redalyc: http://www.redalyc.org/articulo.oa?id=54557477014

Fecha de recepción: 28 Diciembre 2017
Fecha de aprobación: 20 Abril 2018
echa de publicación: 01 Noviembre 2018

\section{Resumen:}

El objetivo es analizar los estudios realizados sobre las personas con discapacidad, desde los enfoques de derechos y capacidades, teniendo en cuenta aspectos de interés de la salud pública como las desigualdades, la justicia social, los determinantes sociales y el bienestar de los colectivos. Se realizó una revisión narrativa de literatura nacional e internacional sobre la discapacidad, a partir de los enfoques de derechos y capacidades. Las búsquedas se llevaron a cabo con palabras claves en español, inglés y portugués. El rastreo de la información arrojó como resultados un total de 32 documentos, sobre los enfoques se encontraron 16 textos referidos a derechos y discapacidad, 11 artículos relacionados con capacidad y discapacidad y 5 artículos que abordan ambos enfoques. Se puede concluir que los enfoques de derechos y capacidades presentan una relación dicotómica y no lineal; incluso respecto a la discapacidad se complementan entre ellos desde diferentes esferas, especialmente para direccionar las barreras que existen entre las personas con discapacidades en los campos de la educación, el empleo y la salud.

Palabras clave: derechos, capacidades, funcionamientos, bienestar, calidad de vida e inclusión social.

\section{Abstract:}

This work aims to analyze the studies conducted on people with disability under the approaches of rights and capabilities considering those aspects interesting in the public health field, such as the inequality, social justice, social determining factors, and well-being of collective groups. A narrative review of the both national and international literature concerning the disability was carried out under both the right approach and the capability approach. Searches were carried out based on keywords in Spanish, English and Portuguese. The quest for information provided 32 documents in total; regarding the approaches under study, 16 papers dealt with rights and disability, 11 papers with capabilities and disability, and 5 papers with both approaches. It can be concluded that the approaches of rights and capabilities have a dichotomous and non-linear relation. Regarding the disability, these two approaches indeed complement each other from their different fields, especially when it comes to cope with the barriers existing between people with disability in the spheres of education, employment and health.

Keywords: rights, capabilities, functioning, well-being, quality of life and social inclusion.

\footnotetext{
a Autor de correspondencia: ngomez@ces.edu.co
} 


\section{Resumo:}

O objetivo é analisar os estudos realizados sobre o pessoal com incapacidade, desde os enfoques de direitos e capacidades, levando em conta aspectos de interesse da saúde pública como as desigualdades, a justiça social, os determinantes sociais e o bem-estar dos coletivos. Realizou-se revisão narrativa de literatura nacional e internacional sobre a deficiência, a partir dos enfoques de direitos e capacidades. As buscas foram levadas a cabo com palavras chave em espanhol, inglês e português. O rastreamento da informação resultou em um total de 32 documentos, sobre os enfoques encontraram-se 16 textos referidos a direitos e deficiência, 11 artigos relacionados com capacidade e deficiência e 5 artigos que abordam ambos os enfoques. Pode-se concluir que as abordagens de direitos e capacidades apresentam uma relação dicotómica e não linear; mesmo respeito da deficiência complementam-se entre eles desde diferentes esferas, especialmente para direcionar as barreiras que existem entre as pessoas com inabilidades nos campos da educação, o emprego e a saúde.

Palavras-chave: direitos, capacidades, funcionamentos, bem-estar, qualidade de vida e inclusão social.

\section{Introducción}

La discapacidad es un concepto pendular y dinámico (1,2). A lo largo del tiempo se han asumido diferentes modelos que actualmente coexisten, entre ellos: el mágico-religioso, el médico o rehabilitador, el social y el biopsicosocial. Sin embargo, en abordaje de las personas con discapacidad ha existido una hegemonía de los modelos médico y social. El primero, asume la discapacidad como un asunto biológico y, en consecuencia, la persona debe ser objeto de rehabilitación. El segundo, plantea que la discapacidad nace de la interacción entre la persona y un medio ambiente particular. El reto está en minimizar las limitaciones o desventajas que tiene la persona con discapacidad en la sociedad a la que pertenece (3).

Con base en los postulados del modelo social, se han implementado acciones positivas e incluyentes. Entre otras, se promulgó la Declaración de Madrid en el año 2002, en la cual se estableció el deber de abandonar las concepciones de sujetos de caridad, pacientes y sujetos con déficits individuales (4). No obstante, se siguió usando algunos términos peyorativos, por ejemplo, el vocablo "discapacitado".

Dentro del modelo social, Kenneth propone una perspectiva universal de la discapacidad. En ella se asume que toda persona está en posición de riesgo, ya que la discapacidad puede presentarse en cualquier etapa de la vida (5). De acuerdo con esta afirmación, la Organización Mundial de la Salud (OMS) señala que todas las personas tendrán una discapacidad temporal o permanente en algún momento de sus vida, y los que sobrevivan y lleguen a la vejez experimentarán cada vez más dificultades para su funcionamiento (6).

Durante el siglo XXI, la discapacidad se ha puesto en la agenda internacional como un problema de salud pública, debido a su incremento mundial. Hoy se estima que el 15\% de la población del planeta tiene alguna y, adicionalmente, peores condiciones de trabajo y salud (7). Para enfrentar las diversas dificultades que enfrentan las personas con discapacidad, se ha señalado que su abordaje sería adecuado y posible desde un enfoque de derechos humanos (8).

En concordancia con lo planteado, la Organización de las Naciones Unidas (ONU) proclamó la Convención de los Derechos de las Personas con Discapacidad en el año 2006, ratificada en el ordenamiento jurídico colombiano en el año 2011. Posteriormente, en septiembre del 2015, los Estados miembros de la ONU firmaron los Objetivos del Desarrollo Sostenible 2015-2030, y en varios apartes quedaron plasmadas las necesidades de las personas con discapacidad. Concretamente, el objetivo 10 propone "Reducir la desigualdad en y entre los países" y, de forma transversal, con el lema "nadie se queda atrás" se pretende la inclusión de los grupos vulnerados (9).

Sobre el enfoque de derechos de las personas con discapacidad, Pérez considera que la citada convención prioriza para su cumplimiento la accesibilidad universal, el diseño universal para todas las personas y los ajustes razonables (10). En relación con el acceso universal, la Convención en su artículo 9..$^{\circ}$ se refiere a "la necesidad de asegurar el acceso de las personas con discapacidad en igualdad de condiciones con las demás, al entorno físico, el transporte, la información y las comunicaciones, incluidos los sistemas y las tecnologías de la información y las comunicaciones, y a otros servicios e instalaciones abiertos al público o de uso público, 
tanto en zonas urbanas como rurales" (11). Por su parte, el diseño universal hace referencia a la elaboración de productos, entornos, programas y servicios que puedan utilizar todas las personas, en la mayor medida posible, sin necesidad de adaptación ni diseño especializado (11).

Los ajustes razonables, en el Artículo 2..$^{\circ}$ aluden a "las modificaciones y adaptaciones necesarias y adecuadas que no impongan una carga desproporcionada o indebida, cuando se requieran en un caso particular, para garantizar a las personas con discapacidad el goce o ejercicio, en igualdad de condiciones con las demás, de todos los derechos humanos y libertades fundamentales" (11). Al respecto, precisa Pérez, el diseño para todos no termina de satisfacer las necesidades de todas las personas con discapacidad, debido a que la casuística es innumerable y no todo puede ser previsto. Por lo que cuando la accesibilidad universal y el diseño para todos fracasa, deben entrar a operar los ajustes razonables (10).

Por otra parte, además de que se ha ampliado considerablemente la protección social a nivel mundial, las personas con discapacidad siguen siendo cinco veces más propensas a incurrir en gastos en salud catastróficos (9). Además, se ha documentado que experimentan desventajas en el ámbito laboral, resultado de las restricciones para acceder a la educación, su reducida movilidad en el entorno y la presencia de otros factores que minan la posibilidad de desempeñarse en una actividad productiva que garantice independencia económica y autonomía personal (12).

Por otra parte, autores como Nussbaum consideran que los problemas de las personas con discapacidad son un asunto no resuelto de las teorías de la justicia social y, concretamente, una deuda del enfoque de capacidades (13). La discapacidad, desde el enfoque de capacidades y funcionamientos, se basa en la libertad de elegir que tienen las personas, y, con base en estos postulados, Sen diferencia los funcionamientos y las capacidades (14).

Los funcionamientos son cosas que las personas logran ser o hacer al vivir, entendidos estos como la situación en que se encuentran respecto a los recursos que adquieren por su trabajo o por el intercambio con otros seres humanos. Por su parte, las capacidades se refieren a las posibilidades que tienen las personas de elegir funcionamientos valiosos para su vida. Asimismo, indica Sen, "la libertad de llevar diferentes tipos de vida se refleja en el conjunto de capacidades de la persona" (15). Luego, sobre estos supuestos, Nussbaum profundiza sobre el concepto de capacidades, ya no como aquellas que solamente se deben elegir, sino como unas mínimas que se deben garantizar: vida, salud corporal, integridad corporal, sentimientos, imaginación y pensamiento, emociones, razón práctica, afiliación, otras especies, capacidad para jugar, control sobre el entorno de cada uno (16).

Concretamente respecto a las personas con discapacidad, Sen explica que presentan una desventaja en términos de libertad para vivir una vida buena. Dicha desventaja se relaciona, entre otros, con deterioro de la capacidad para obtener ingresos; de hecho, están dentro de los seres humanos más pobres del mundo y los más desatendidos (14).

El objetivo es analizar los estudios realizados sobre las personas con discapacidad, desde los enfoques de derechos y capacidades, teniendo en cuenta aspectos de interés de la salud pública como las desigualdades, la justicia social, los determinantes sociales y el bienestar de los colectivos.

En este análisis la salud pública se entiende como "un campo transdisciplinar organizado en torno a un conjunto de saberes y prácticas derivadas de distintas procedencias disciplinares y sociales, tendientes al entendimiento de la salud y el bienvivir humano y al desarrollo de acciones sociales, institucionales, profesionales y políticas orientadas a la promoción de la salud, la prevención de la enfermedad y la superación de barreras (sociales, políticas, culturales, económicas que impiden a las personas, ya los colectivos humanos lograr una vida digna" (17). 


\section{Metodología}

Este artículo corresponde a una revisión narrativa. Este tipo de revisión consiste en una síntesis narrativa y comprensiva de información publicada previamente. Estas revisiones pueden motivar una discusión, una idea de investigación, o aclarar algunos conceptos (18).

Para dar alcance al objetivo del artículo, se realizó una revisión de literatura nacional e internacional sobre las personas con discapacidad desde los enfoques de derechos y capacidades. La revisión incluyó la consulta bibliográfica de artículos, libros electrónicos y documentos institucionales publicados en bases de datos como PubMed, EBSCO, Redalyc, Scielo y el motor de búsqueda Scholar Google. Las búsquedas fueron realizadas con palabras clave en español, inglés y portugués. Los términos utilizados fueron discapacidad, discapacitados, deficiencias, enfoque de derechos, enfoque de capacidades, capacidades y funcionamientos. En la pesquisa se tuvo en cuenta artículos de enfoques cualitativos, cuantitativos y mixtos.

Los datos recolectados de las diferentes fuentes de información fueron analizados y organizados según su cronología y consultados diferentes campos del conocimiento como la salud pública, la medicina, las ciencias sociales, la psicología, la filosofía, entre otros, a través de una matriz en Excel por año, título, tipo, revista, enfoque y área de conocimiento.

Las revistas en las cuales se encontró la información requerida fueron las siguientes: Ciência \& Saúde Coletiva, Revista Chilena de Salud Pública, Revista de Salud Pública de la Universidad Nacional de Colombia, Revista ius et práxis, Boletín Mexicano de Derecho Comparado, Revista de Direitos e Garantias Fundamentais, American University International Law Review, Revista da AJURIS, Fordham Law Review, Derechos Humanos y Discapacidad, Civilizar, Ciencias Sociales y Humanas, Convergencia Revista de Ciencias Sociales, Revista Direitos Sociais e Politicas Públicas (UNIFAFIBE), Disability And Society, Journal of Disability Policy Studies, FMC - Formación Médica Continuada en Atención Primaria, Revista de Psiquiatría y Salud Mental, Revista Médica Clinica Las Condes, Australian Occupational Therapy Journal, Revista Chilena de Terapia Ocupacional, Revista Internacional de Filosofía, Revista de Estudiantes de Filosofía, Revista Iberoamericana de Filosofía, Politica y Humanidades, Universidad Autónoma del Estado de México, SUR-International Journal on Human Rights, Norte de Salud mental, Revista Colombiana de Bioética.

El proceso de síntesis documental se realizó a partir del análisis de los datos objeto de la revisión. Las categorías emergentes que ordenaron la información a partir de las palabras claves fueron: modelos de la discapacidad, salud de las personas con discapacidad, enfoque de derechos y enfoque de discapacidad identificados en la matriz; además, se agregó el análisis de la relación con la salud pública a cada documento. Las conclusiones resultaron de un proceso analítico y crítico de la información extractada y sintetizada de las fuentes. Finalmente, se realizaron recomendaciones y una relación bibliográfica.

\section{Resultados y discusión}

La búsqueda de la información arrojó como resultados 32 documentos distribuidos de la siguiente forma: veinte artículos de reflexión, cuatro artículos resultados de investigaciones, tres artículos de revisión, tres libros electrónicos de expertos y dos informes o investigaciones de organismos multilaterales. Sobre los enfoques se rastrearon dieciséis artículos referidos a derechos y discapacidad, once artículos relacionados con capacidad y discapacidad y cinco artículos que abordan ambos enfoques.

La presentación de los resultados está organizada por los ejes conceptuales abordados en las publicaciones relacionadas a partir de las categorías emergentes. Desde el enfoque de derechos se encontraron textos respecto a los modelos de discapacidad médico y social, discusiones sobre la salud de las personas con discapacidad y sobre normas de protección de las personas con discapacidad. Con relación al enfoque de capacidades se rastrearon documentos sobre las personas con discapacidad y enfoque de capacidades y se 
evidenciaron estudios que analizaban las personas con discapacidad desde ambos enfoques, capacidades y derechos.

En la tabla 1 se presentan los resultados y las discusiones planteadas y su relación con la salud pública.

\section{Las personas con discapacidad desde el enfoque de derechos}

TABLA 1

Estudio de los modelos de discapacidad y el enfoque de derechos

\begin{tabular}{|c|c|c|c|}
\hline TITULO ARTICULO & ANNO & REVISTAINSTITUCION & CANIPO DE CONOCIIIENTO \\
\hline $\begin{array}{l}\text { La extensión de los } \\
\text { ajustes razonables en } \\
\text { el derecho de las } \\
\text { personas en situa ciön } \\
\text { de discapacidad de } \\
\text { acuerdo al enfoque } \\
\text { social de derechos } \\
\text { humanos 19) }\end{array}$ & 2016 & Revista ius et praxis & Juridico \\
\hline $\begin{array}{l}\text { A abordagem da } \\
\text { deficiência em face } \\
\text { da expansão dos } \\
\text { direitos humanos } \\
\text { (20) }\end{array}$ & 2016 & Revista de Direitos e Garantias Fundamentais & Juridico \\
\hline $\begin{array}{l}\text { Discapacidady } \\
\text { mendicidad enla era } \\
\text { de la } \\
\text { Convención: ¿postal } \\
\text { del pasado? (21) }\end{array}$ & 2015 & Convergencia Revista de Ciencias Sociales & Ciencias Sociales \\
\hline $\begin{array}{l}\text { Hacia un modelo de } \\
\text { atención a la } \\
\text { discapacidadbasado } \\
\text { en los derechos } \\
\text { humanos (22) }\end{array}$ & 2013 & Boletin Mexicano de Derecho Comparado & Juridico \\
\hline $\begin{array}{l}\text { Perfil daspessoas } \\
\text { com defciência fisica } \\
\text { e Políticas Públicas: }\end{array}$ & 2007 & Derechos Humanos y Discapacidad & Ciencias Sociales \\
\hline $\begin{array}{l}2 \text { distância entre } \\
\text { intenções e gestos } \\
\text { (23) }\end{array}$ & & & \\
\hline
\end{tabular}

Fuente: elaboración propia a partir del rastreo realizado. 
De Menezes (20), Victoria (22), Palacio (24) y De Asís (23) hacen disertaciones sobre los cambios de paradigma para el abordaje de las personas con discapacidad con la implementación del modelo social. Sobre ello resaltan que el enfoque de derechos humanos centra su discurso en la persona y su dignidad. Finsterbusch (19), por su parte, señala la necesidad de realizar ajustes razonables y advierte que es difícil su afectividad e importante ir construyéndolos a partir de los referentes que existan y se acepten, en beneficio de las personas con discapacidad.

En la mayoría de las publicaciones se hace énfasis en que las dificultades de esta población se presentan en las barreras a la participación y a la igualdad. Un caso concreto se refiere a los procesos sociales que conducen al ejercicio de la mendicidad en varones con discapacidad de la ciudad de Buenos Aires, con la intención de problematizar sobre la necesidad de un cambio en las actitudes hacia esta minoría a partir de la Convención internacional sobre los derechos de las personas con discapacidad y la necesidad de aplicar el enfoque de derechos (21).

En relación con la salud pública, la mayoría de los textos abordan el tema de desigualdades respecto a las personas con discapacidad. Reconocen que son un grupo vulnerable y plantean la importancia del enfoque de derechos para lograr la igualdad y eliminar las barreras que les impone la sociedad (19, 20, 21, 22). Respecto a las desigualdades, Finsterbusch estudia los ajustes razonables como una solución ante las desventajas y la discriminación que pueden darse por su estado de salud, bajo nivel educación o requerimientos especiales para desempeñarse en el trabajo (19). De Menezez destaca la necesidad de medidas para lograr la igualdad jurídica, de trato y oportunidades en aras de la inclusión social y una vida independiente (20). Ferrante precisa que las medidas tienden a generar un discurso de buenas intenciones y a invisibilizar la proliferación de modos de desigualdad (21). Por su parte, Victoria se centra en el derecho como un instrumento internacional para generar actitudes tolerantes e inclusivas para que las personas con discapacidad logren la autonomía personal, no sean discriminadas, cuenten con accesibilidad universal, se normalice el entorno y exista diálogo social (22).

En general, se encuentra que hay convergencia en las discusiones que se dan respecto a la necesidad de lograr la igualdad para las personas con discapacidad y hay una línea clara que pretende orientar los contextos históricos y sociales para afrontar los problemas de justicia social que presentan en los diferentes ámbitos de la vida diaria.

Por otra parte, desde una perspectiva de la salud pública, en su dimensión de campo de conocimiento (25), los estudios de los modelos de la discapacidad con enfoque de derechos abordan aspectos teóricos a partir de diferentes disciplinas, para tratar los problemas de discriminación, exclusión y desigualdad de las personas con discapacidad y, por tanto, demandan más investigación, discusión y sistematización. Es decir, requieren mayor orientación a la aplicación del enfoque a través de acciones concretas por parte del Estado y la academia.

Por otra parte, la salud de las personas con discapacidad, de acuerdo con el enfoque de derechos, se presenta como un tema relevante en materia de salud pública, como se explica en la tabla 2. 
TABLA 2

La salud de las personas con discapacidad desde el enfoque de derechos

\begin{tabular}{|c|c|c|c|}
\hline TITULO ARTICULO & ANO & REVISTA/INSTITUCION & $\begin{array}{c}\text { CAMPO DE } \\
\text { CONOCIMIENTO }\end{array}$ \\
\hline $\begin{array}{l}\text { Perfil das pessoas com deficiência } \\
\text { física e Políticas Públicas: } \\
\text { a distância entre intenções e gestos } \\
\text { (26) }\end{array}$ & 2016 & Ciência \& Saúde Coletiva & Salud pública \\
\hline $\begin{array}{l}\text { La Convención sobre los derechos } \\
\text { de las personas con discapacidad en } \\
\text { salud mental. Una cuestión de } \\
\text { derechos humanos (27) }\end{array}$ & 2016 & $\begin{array}{l}\text { Revista de Psiquiatriay Salud } \\
\text { Mental }\end{array}$ & Medicina \\
\hline $\begin{array}{l}\text { Primera infancia y discapacidad en } \\
\text { Chile. Revisión y enfoque actual a } \\
\text { los programas de gobiemo (28) }\end{array}$ & 2015 & $\begin{array}{l}\text { Revista Médica Clínica Las } \\
\text { Condes }\end{array}$ & Medicina \\
\hline $\begin{array}{l}\text { El enfoque de derechos en la } \\
\text { política laboral y salarial: } \\
\text { construcción de un marco } \\
\text { metodológico para aplicarse en } \\
\text { Costa Rica, El Salvador, } \\
\text { Guatemala, Nicaragua y México } \\
\text { (29) }\end{array}$ & 2013 & Cepal & Economía \\
\hline
\end{tabular}

Fuente: elaboración propia a partir del rastreo realizado.

Inicialmente, el rastreo de los estudios de la salud de las personas con discapacidad desde la perspectiva del enfoque de derechos arroja que los autores se centran en tipos de discapacidades como física o mental y también estudian subgrupos como mujeres, niños y adultos mayores.

Cavalheiro analiza la discapacidad física y el perfil epidemiológico de una comunidad. Asimismo, advierte la distancia que hay entre las políticas públicas existentes que acogen el enfoque de derechos y las realidades de la población. Además, señala que es necesario trabajar en la prevención de enfermedades crónicas no transmisibles, la adopción de prácticas para disminuir los accidentes de tránsito y otras que permitan el acceso al trabajo, la educación y el ocio para esta población. Adicionalmente, indica que se requieren 
intervenciones para eliminar las barreras tanto ambientales como sociales. También advierte que las personas con discapacidad física son más vulnerables al desarrollo de problemas de salud por el contexto social (26).

En esta línea, también se encontró un estudio realizado por la Cepal en el que se muestra que en países de América Latina hay avances en materia de política de empleo para los grupos vulnerados; en el caso de las personas con discapacidad, basados en la nueva concepción de la Convención de las Personas con Discapacidad, pero aún se presentan violaciones de derechos (29). Concretamente, este organismo multilateral hace una relación entre trabajo y salud de las personas con discapacidad y señala que el derecho a la salud comprende una gran variedad de factores socioeconómicos que permiten que lleven una vida sana, considerando factores determinantes básicos: alimentación, nutrición, vivienda, acceso a agua potable y condiciones sanitarias adecuadas, de trabajo seguros y un medio ambiente sano (29).

Desde una mirada de la salud pública, ambos estudios, los realizados por Cavalheiro y el de la Cepal, reconocen la relevancia de los determinantes sociales en el proceso salud-enfermedad de las personas con discapacidad, en el entendido de que son socialmente desfavorecidas porque tienen menos acceso a las recursos sanitarios, se enferman y mueren antes que otras personas que tienen una posición social más privilegiada (30). Ello no implica, sin embargo, que no se existan personas con discapacidad con recursos económicos, pero las barreras que enfrentan quienes no los tienen se maximizan por las dificultades para acceder, por ejemplo, a educación y salud.

Ramos, por su parte, hace una discusión sobre la protección de los derechos humanos de las personas con discapacidad mental en caso de hospitalizaciones involuntarias, esterilización y la necesidad de revisar las leyes al respecto. Así, plantea las discusiones de personas con discapacidades mentales graves, especialmente respecto a su derecho a una vida digna, calidad de vida y la capacidad suficiente para tomar decisiones que hacen que se tenga que resolver con la mejor interpretación de la voluntad (27).

En Colombia, este es realmente un problema de salud pública que afecta a esta población y tiene un impacto social ante las barreras que se imponen especialmente en materia de empleo. Las personas con discapacidad son obligadas a realizar procesos judiciales en los cuales se nombra un representante para tomar decisiones por ellos, conforme al Código Civil colombiano (31). La posibilidad de evitarlo se lograría a través de normas que permitan la aplicación de la Convención de las Personas con Discapacidad respecto a la decisión con apoyo (11), es decir, eliminando la sustitución o subrogación de la capacidad legal actual y facilitando el acompañamiento en la toma de decisiones.

Para el enfoque de derechos, tiene especial relevancia lo relacionado con niños y niñas, grupo vulnerable de interés de la salud pública (32). Desde esta óptica, Sánchez hace una revisión acerca de la primera infancia en situación de discapacidad en Chile, aborda los programas de gobierno que existen, su actual enfoque de derechos, e indica que se va en camino de la igualdad de oportunidades, acceso oportuno y calidad en salud, desarrollo pleno y digno a través de las propuestas existentes en materia de rehabilitación, políticas públicas, proyectos de ley para acceso a medicamentos, entre otras (28).

Hasta ahora es visible la preocupación por las acciones que se implementan para abordar los problemas de salud que afrontan las personas con discapacidad, desde diferentes disciplinas y, especialmente, en lo que tiene que ver con la atención en salud. No obstante, falta profundizar en la afectación de los determinantes sociales que se citan respecto a un proceso social. Por otra parte, solamente en uno de los documentos (26) hay reconocimiento expreso de que la salud de las personas con discapacidad es un problema de la salud pública que requiere intervenciones a partir diferentes campos del conocimiento.

Sobre el tema legislativo, es importante analizar, desde el enfoque de derechos, las normas de protección para las personas con discapacidad, como se en la tabla 3. 
TABLA 3

Las normas de protección para las personas con discapacidad desde el enfoque de derechos

\begin{tabular}{|c|c|c|c|}
\hline TIIULO ARTICULO & AÑO & REVISTAINSTITUCION & $\begin{array}{c}\text { CAMPO DE } \\
\text { CONOCIIIENTO }\end{array}$ \\
\hline $\begin{array}{l}\text { Informe altemativo de la coalición } \\
\text { colombiana para la implementación } \\
\text { de la Convención sobre los } \\
\text { derechos de las personas con } \\
\text { discapacidad(33) }\end{array}$ & 2016 & ONU & Ciencias Sociales \\
\hline $\begin{array}{l}\text { Igual reconocimiento de las } \\
\text { personas con discapacidad arte la } \\
\text { ley. Algunos comentarios sobre la } \\
\text { "Convención sobre los derechos de } \\
\text { las personas con discapacidad" (34) }\end{array}$ & 2016 & $\begin{array}{l}\text { FMC - Formación Médica } \\
\text { Continuada en Atención Primaria }\end{array}$ & Medicina \\
\hline $\begin{array}{l}\text { Análisis desde el enfoque de } \\
\text { derechos a la política chilena en } \\
\text { discapacidadmental (35) }\end{array}$ & 2015 & Revista Chilena de Salud Pública & Salud Pública \\
\hline $\begin{array}{l}\text { A efetiva ção dos direitos das } \\
\text { pessoas com deficiência pelos } \\
\text { sistemas de proteção dos direitos } \\
\text { humanos: sistema americano e } \\
\text { europeu(36) }\end{array}$ & 2013 & $\begin{array}{l}\text { Revista Direitos Sociaise } \\
\text { Politicas Puiblicas (CNIFAFIBE) }\end{array}$ & Ciencias Sociales \\
\hline $\begin{array}{l}\text { Principios bioéticos y discapacidad } \\
\text { la perspectiva de su inclusión en las } \\
\text { politicas públicas ( } 37 \text { ) }\end{array}$ & 2011 & Revista Colombiana de Biośtica & Bioética \\
\hline $\begin{array}{l}\text { Los derechos de las personas con } \\
\text { discapacidad( }(8)\end{array}$ & 2008 & Universidad de Buenos Aires & Ciencias Sociales \\
\hline $\begin{array}{l}\text { Enfoque de derechos humanos } \\
\text { en la politica pública de } \\
\text { discapacidad(38) }\end{array}$ & 2007 & $\begin{array}{l}\text { Civilizar. Ciencias Socialesy } \\
\text { Humanas }\end{array}$ & Ciencias Sociales \\
\hline
\end{tabular}

Fuente: elaboración propia a partir del rastreo realizado.

Como punto en común en los textos $(33,34,35,36)$ se discute la aplicación efectiva de las normas existentes en diferentes legislaciones para la población con discapacidad, con posterioridad a la promulgación de la Convención de los derechos de las personas con discapacidad. Pelli sostiene que la protección de los derechos humanos tiene que darse tanto en el ámbito nacional como en el internacional (36). 
En conexión con la salud pública, en las discusiones del enfoque de derechos en las normas relacionadas con las personas con discapacidad, se abordan temas como su calidad de vida, las falencias de los sistemas de salud para atenderlas y la necesidad de políticas públicas que favorezcan su inclusión social (8).

La coalición colombiana para la implementación de la citada convención se ha manifestado en relación con el incumplimiento de este instrumento internacional. Afirma que existen normas en materia de salud, pero a las personas con discapacidad les falta más accesibilidad a los servicios y no gozan de una óptima e integral atención que impacte positivamente su calidad de vida, lo que afecta la inclusión social efectiva (33).

Sobre la igualdad ante la ley como un principio básico de la protección de los derechos humanos, Ramos plantea su preocupación por las personas con discapacidad mental y la necesidad de tratamiento oportuno, rehabilitación e integración a la sociedad (34). Al respecto, Pino también indica que es necesario materializar el enfoque de derechos en el ámbito de las políticas públicas para lograr un impacto real en este colectivo (35).

En relación con políticas públicas, Rozo plantea que se requiere mejorar las condiciones de vida de las personas con discapacidad, ya que históricamente han sido excluidas y esto disminuye sus oportunidades de participación social y ciudadana (37). En este sentido, Dussan considera que un punto crítico de las políticas públicas para abordar los problemas de la población con discapacidad es la ausencia de acciones estatales y afirma que es necesario articularlas con los Objetivos del Milenio para mejorar sus condiciones de vida (38).

Por último, Eroles sostiene que las normas que garantizan la aplicación de la Convención de las personas con discapacidad representan la lucha contra la exclusión y la desigualdad. Advierte que es necesario construir una democracia para todos que garantice la participación plena y la calidad de vida de las personas con discapacidad. En su trabajo sobresale la preocupación por los referentes normativos que se requieren en materia de salud mental y por lograr el más alto nivel posible de salud (8).

Un eje relevante en las tres perspectivas analizadas: modelos hegemónicos, salud y normativa, es que la igualdad se entiende desde el enfoque de derechos humanos, en el sentido de que todos somos iguales en dignidad, libertad y derechos, sin distinción alguna (8), y se tiene como premisa la justicia social respecto a un grupo socialmente vulnerable denominado personas con discapacidad. 


\section{Las personas con discapacidad desde el enfoque de capacidades}

TABLA 4

El enfoque de capacidades y la discapacidad

\begin{tabular}{|c|c|c|c|}
\hline TÍTULO ARTÍCULO & Año & REVISTA/LSTITUCIÓN & CAMPO DE CONOCDIIENTO \\
\hline $\begin{array}{l}\text { La protección de las personas con discapacidad en la Convención Americana sobre Derechos } \\
\text { Humanos desde el enfoque de las capacidades: de la igualdad de trato a la igualdad de } \\
\text { oportunidades (39) }\end{array}$ & 2015 & $\begin{array}{l}\text { American University } \\
\text { International Law Review }\end{array}$ & Juridico \\
\hline Quality of life, participation and occupational rights: A capabilities perspective (40) & 2015 & $\begin{array}{l}\text { Australian Occupational } \\
\text { Therapy Joumal }\end{array}$ & Medicina \\
\hline An extension of the capability approach: Towards a theory of dis-capability (41) & 2011 & $\begin{array}{l}\text { ALTER, European Joumal } \\
\text { of Disability Research }\end{array}$ & Interdisciplinaria \\
\hline $\begin{array}{l}\text { Justicia, igualdad, discapacidad: una reflexión desde el "enfoque de las capacidades" y la teoria de } \\
\text { la justicia de John Rawls (42) }\end{array}$ & 2014 & $\begin{array}{l}\text { Revista Chilena de Terapia } \\
\text { Ocupacional }\end{array}$ & Medicina \\
\hline $\begin{array}{l}\text { ¿Hacia una justicia sin fronteras? El enfoque de las capacidades de Martha Nussbaum y los límites } \\
\text { de la justicia (43) }\end{array}$ & 2013 & $\begin{array}{l}\text { Revista Internacional de } \\
\text { Filosofia }\end{array}$ & Filosofia \\
\hline $\begin{array}{l}\text { Valoración ocupacional de las personas con discapacidad, desde la perspectiva de las capacidades } \\
\text { humanas (44) }\end{array}$ & 2013 & Revista de Salud Pública & Salud Pública \\
\hline $\begin{array}{l}\text { Human diversity and asymmetries: a reintempetation of the social contract under the capabilities } \\
\text { approach (45) }\end{array}$ & 2011 & $\begin{array}{l}\text { SUR-International Jownal } \\
\text { on Human Rights }\end{array}$ & Derechos humanos \\
\hline El "enfoque de las capacidades" de Nussbaum y el concepto de "discapacidad" (46) & 2009 & $\begin{array}{l}\text { Revista de Estuatiantes de } \\
\text { Filosofia }\end{array}$ & Filosofia \\
\hline La discapacidad dentro del enfoque de capacidades y funcionamientos de Amartya Sen (47) & 2008 & $\begin{array}{l}\text { Revista Iberoamericana de } \\
\text { Filosofia, Pollticay } \\
\text { Humanidades }\end{array}$ & Filosofia \\
\hline The capability approach and disability (48) & 2006 & $\begin{array}{l}\text { Jownal of Disability Policy } \\
\text { Studies }\end{array}$ & Ciencias Sociales \\
\hline Capabilities and disability: the capabilities framework and the social model of disability (49) & 2004 & Disability and Society & Ciencias Sociales \\
\hline
\end{tabular}

Fuente: elaboración propia a partir del rastreo realizado.

El tema del enfoque de capacidades y la relación con las personas con discapacidad tiene como presupuesto los postulados de Sen y Nussbaum, con especial énfasis en las discusiones de esta última autora, quien en sus obras insiste en que se trata de uno de los problemas no resueltos de la justicia social. Un número importante de artículos atribuyen al enfoque una especial utilidad para la interpretación de la Convención internacional sobre los derechos de las personas con discapacidad, mejorando sus derechos y dignidad (39, 42, 43, 45, 46, 47, 48, 49, 50).

Es importante señalar que a partir de la concepción del enfoque de capacidades abordado en los textos revisados, se encuentra una relación estrecha con la salud pública desde su perspectiva de conjunto de acciones individuales y colectivas, públicas y privadas para hacer posible el bienestar y el bien vivir humano (25). En este sentido, se observa a las personas con discapacidad a partir de sus determinantes sociales, calidad de vida, 
estilos de vida y, en general, el bienestar que se pretende para lograr una sociedad más justa con participación social $(39,40,41,42,43,44,45,46,47,48,49)$.

El estudio de Torres indica que si bien el enfoque de las capacidades mejora las posibilidades de las personas con discapacidad respecto a sus derechos y su dignidad, hay un problema profundo, ya que muchas de ellas no desean perseguir el despliegue de sus capacidades básicas. En este sentido, el autor interpreta que el desarrollo de capacidades es un asunto que deben resolver las persona con discapacidad, desconociendo lo planteado por Nussbaum respecto a que las capacidades básicas no solamente se deben ofrecer, sino garantizar (42).

Por otra parte, hay dos estudios relacionados con el trabajo, el primero esboza la relevancia del enfoque de capacidades, a partir de Sen, para terapias ocupacionales que tratan de abordar las desigualdades de oportunidad ocupacional e inequidades en la participación (40). Y el segundo presenta una herramienta denominada "valoración ocupacional de las personas con discapacidad en Colombia", que tiene en cuenta los elementos de capacidades y funcionamientos de este enfoque (44). Si bien en los textos se habla del enfoque de capacidades, también se hace énfasis en el tema de habilidades, especialmente aquellas que favorezcan la productividad y no la capacidad de elegir la vida que quieren llevar las personas con discapacidad.

Otros textos profundizan en la discapacidad como una categoría inmersa en el enfoque de capacidades y hacen énfasis en conceptos desarrollados por Sen, como las agencias, precisando que cuando las personas adquieren capacidades, alcanzan agencias, entendidas como la habilidad de definir las metas propias de forma autónoma y actuar a partir de estas, lo cual se da en ejercicio de la libertad de ser agente, es decir, cuando se es libre de hacer y conseguir los propósitos que se consideran importantes $(45,47,48,49)$.

Asimismo, analizan la relación entre libertad de agencia y libertad de bienestar. Toboso, especialmente, señala que esta última se refiere a la libertad de conseguir aquellas cosas que constituyen su propio bienestar y, por tanto, es la que mejor refleja las capacidades de una persona, ya que la libertad solamente de agencia puede llevar a tomar decisiones que no vayan en beneficio del propio bienestar. En su trabajo se resalta la importancia de lograr, a través de este enfoque, la calidad de vida de las personas con discapacidad, y dentro de los modelos sociales acoge la tesis de la diversidad funcional, en el entendido de que no son diversas las personas, sino su manera particular de lograr idénticos funcionamientos (47). En relación con la salud pública, este autor también analiza los procesos salud-enfermedad de las personas con discapacidad desde una perspectiva de los estados de salud propuesta por la OMS, en particular porque tiene en cuenta los factores contextuales: ambientales y personales, que inciden en su estilo de vida (47).

Mitra, con una mirada económica, se refiere a las estimaciones actuales de la calidad de vida de los hogares con personas con discapacidad, por las dificultades que pueden presentar para obtener ingresos y conseguir empleo. Así, afirma que con base en el enfoque de capacidades se pueden estudiar las características personales de este grupo de personas, sus recursos y su entorno (48). Otro aspecto abordado del enfoque de capacidades corresponde a la garantía de los medios y los recursos para que las personas con discapacidad puedan ejercer sus derechos y participar en la formulación de políticas públicas. En este sentido, Reicher considera que el enfoque de capacidades permite el análisis de la diversidad humana, la discapacidad y la participación para lograr una sociedad más justa (45).

De forma contraria, autores como Bellanca entienden que dentro del enfoque de derechos las personas con discapacidad son seres con capacidades limitadas respecto a sus objetivos, ambiciones y sistemas de valores (41). Al respecto, es importante precisar que esta postura es una tesis minoritaria en relación con los demás textos rastreados en esta revisión. 


\section{Las personas con discapacidad desde ambos enfoques}

TABLA 5

Abordaje de la discapacidad desde los enfoques de derecho y capacidades de forma simultánea

\begin{tabular}{|c|c|c|c|}
\hline TITULO ARTÍCULO & AÑo & REVISTA/INSTITUCIÓN & CAMPO DE INFORMACIÓN \\
\hline $\begin{array}{l}\text { Necesidades capacidadesy } \\
\text { derechos humanos bajo la } \\
\text { perspectiva de Martha } \\
\text { Nussbaum(51) }\end{array}$ & 2016 & $\begin{array}{l}\text { Universidad Autónoma del Estado de } \\
\text { México }\end{array}$ & Filosofia \\
\hline $\begin{array}{l}\text { Direitos humanos } \\
\text { fundamentais das pessoas } \\
\text { com deficiências: the } \\
\text { capability approach }(50)\end{array}$ & 2014 & Revista da AJURIS & Jurídico \\
\hline $\begin{array}{l}\text { El modelo social de } \\
\text { discapacidad: } \\
\text { orígenes, caracterizacióny } \\
\text { plasmación } \\
\text { en la Convención } \\
\text { Intemacional } \\
\text { sobre los Derechos de las } \\
\text { Personas } \\
\text { con Discapacidad(24) }\end{array}$ & 2008 & CERMI & Jurídico \\
\hline $\begin{array}{l}\text { Discapacidady derechos } \\
\text { humanos (52) }\end{array}$ & 2010 & Norte de Salud Mental & Psicología \\
\hline $\begin{array}{l}\text { Capabilities and human } \\
\text { rights (53) }\end{array}$ & 1997 & Fordham Law Review & Jurídico \\
\hline
\end{tabular}

Fuente: elaboración propia a partir del rastreo realizado.

Desde la perspectiva de la justicia coexisten dos conceptos relevantes para la discusión: derechos y capacidades. Lo primero que debe advertirse es que no son sinónimos. Derechos se refiere a aquellos que son inherentes al ser humano (con independencia de su reconocimiento en las normas) y las capacidades a la libertad de elegir funcionamientos valiosos (54). Sin embargo, el escenario de encuentro de ambos enfoques es la justicia social, en el sentido que buscan la equidad y la igualdad (55). 
Sobre esta relación de los enfoques de derechos y capacidades respecto a las personas con discapacidad, según Nussbaum, son convergentes de dos formas. La primera, reconociendo los derechos como capacidades combinadas (el derecho a la participación política, el derecho al libre ejercicio religioso, la libertad de expresión, entre otros. La segunda, como las citadas “capacidades básicas", ya que por lo general se cree que los derechos humanos se derivan de alguna característica propia del ser humano, siendo su postura final asumir los derechos como capacidades combinadas (53).

Zetina concluye que un correcto funcionamiento de los derechos humanos puede ayudar sustancialmente a que el enfoque de las capacidades sea puesto en marcha favorablemente, sobre todo para dar respuesta a la deuda social que se tiene con las personas con discapacidad (51). Por su parte, Strapazzon estudia la relación del modelo social de la discapacidad con el enfoque de capacidades, para atender las desigualdades y las exclusiones a partir del reconocimiento y el respeto de los derechos humanos de las personas con discapacidad (50). Igualmente, Palacio precisa aspectos relevantes sobre los derechos de las personas con discapacidad y la relación con la calidad de vida (24).

Por su parte, Urmeneta cita las capacidades como un modelo que va más allá de los derechos humanos, puesto que promulga derechos justos (52). Sobre el término modelo, sus propios autores reconocen que es un enfoque, y respecto a la connotación de justos, los derechos deben serlo porque son inherentes al ser humano.

En este aparte es evidente que los documentos revisados, en general, siguen las líneas de los demás resultados de esta búsqueda sobre los ejes que se deben fortalecer para lograr desde los enfoques de derechos y capacidades el bienestar de las personas con discapacidad.

\section{Conclusiones}

A partir de los enfoques hegemónicos, es decir, el médico y el social, hay convergencia en las discusiones que se dan respecto a la igualdad, la dignidad, la libertad y los derechos, sin distinción alguna de las personas con discapacidad. Además, hay una línea clara que pretende orientar los contextos históricos y sociales para afrontar los problemas de justicia social que presentan en los diferentes ámbitos de la vida diaria.

Con referencia a la salud de las personas con discapacidad, se puede evidenciar que hay una preocupación por las acciones que se implementan a partir de diferentes disciplinas; no obstante, se requiere una articulación para brindar soluciones efectivas desde el enfoque de derechos, que no se limiten a la prestación de servicios. En este sentido, falta profundizar en la afectación de los determinantes sociales orientados al enfoque de derechos humanos.

En el contexto normativo para la protección de las personas con discapacidad, se requiere que el enfoque de derechos, además de quedar plasmado en los instrumentos nacionales e internacionales, se cumpla y materialice en acciones concretas para esta población.

En relación con el enfoque de capacidades, puede decirse que es un desarrollo del enfoque de derechos que pretende el bienestar de las personas y reconoce la vulnerabilidad de grupos minoritarios como las personas con discapacidad. Para lograr la justicia social, es necesario brindar garantías respecto a los medios y recursos que se requieren para ejercer sus derechos y lograr una participación en los procesos sociales.

En cuanto al enfoque de derechos de las personas con discapacidad, este reconoce su vulnerabilidad social $(19,20,21,22)$ y sus condiciones de desigualdad (19) y discriminación, especialmente en materia de salud (19) y empleo (30). En este sentido, se han direccionado los estudios y se ha profundizado especialmente en los temas de empleo, sistema de salud, calidad de vida y salud mental. En los documentos rastreados, es relevante la importancia de las políticas públicas para este grupo poblacional.

Por su parte, desde la perspectiva de la salud pública, el enfoque de capacidades también considera importantes las acciones individuales y colectivas, públicas y privadas, para hacer posible el bienestar y el bien vivir humano de las personas con discapacidad, en el sentido que reconoce que se trata 
trata de un asunto que, más allá de las deficiencias físicas, psíquicas y sensoriales, y respecto a las barreras para la participación y la igualdad, tiene en cuenta la importancia de garantizar la calidad de vida y en general el bienestar a estas grupo vulnerado socialmente.

Los enfoques de derechos y capacidades presentan una relación dicotómica y no lineal respecto a la discapacidad, ya que se complementan en diferentes esferas para atender las barreras de esta población en materia de educación, empleo y salud. Adicionalmente, ambos enfoques contribuyen a las problemáticas de salud pública que presentan las personas con discapacidad, las cuales son abordadas desde las desigualdades, la justicia social, los determinantes sociales y el bienestar de los colectivos humanos.

Finalmente, esta revisión narrativa evidencia que sobresale la literatura teórica y se identifica una mayor necesidad de aplicación empírica que contribuya, desde una mirada salubrista, a resolver los problemas reales de esta población.

\section{Referencias bibliográficas}

1. López M. Modelos teóricos e investigación en el ámbito de la discapacidad. Hacia la incorporación de la experiencia personal. En: Docencia e investigación: año XXXI [Internet]. Toledo: Escuela de Magisterio de la Universidad de Castilla-La Mancha; 2016 [citado 2017 abr. 15]. p. 2015-240. Disponible en: https://ruidera.uclm.es/xmlu i/handle/10578/8063

2. Moreno M. Infancia, políticas y discapacidad [Internet]. Bogotá: Universidad Nacional de Colombia; 2011. Disponible en: https://www.bdigital.unal.edu.co/3597/4/Libro_Infancia_Politicas_Discapacidad.pdf

3. Padilla A. Discapacidad: contexto, concepto y modelos. Int Law [Internet]. 2010 [citado 2015 abr. 12];16:381-414. Disponible en: https://www.scielo.org.co/scielo.php?pid=S1692-81562010000100012\&script=sci_arttext\&t $\operatorname{lng}=\mathrm{es}$

4. Congreso Europeo sobre las personas con discapacidad. Declaración de Madrid "No discriminación más acción positiva es igual a inclusión social”. 2002 [citado 2015 abr. 5]; Disponible en: https://civegi.uji.es/isonomia/do cs/spanish/orientacion/webs/declaracionmadrid.pdf

5. Irving K. Toward the necessary universalizing of a disability policy. Milbank Q [Internet]. 1989;67:401-28. Disponible en: https://www.jstor.org/stable/3350151

6. Organización Mundial de la Salud, Banco Mundial. Informe mundial sobre discapacidad [Internet]. Ginebra, Suiza: OMS; 2011.p. 388. Disponible en: https://www.who.int/disabilities/world_report/2011/es/

7. Organización Mundial de la Salud. Plan de acción mundial de la Organización Mundial de la Salud sobre discapacidad 2014-2021: mejor salud para todas las personas con discapacidad [Internet]. Ginebra: Organización Mundial de la Salud; 2014 [citado 2015 abr. 13] p. 23. Report No.: A67. Disponible en: https://www.who.int /disabilities/policies/actionplan/disability_action_plan_es.pdf

8. Eroles C, Fiamberti H. Los derechos de las personas con discapacidad [Internet]. Universidad de Buenos Aires. Argentina; 2002. Disponible en: https://www.cud.unlp.edu.ar/uploads/docs/libro_eroles_fiamberti.pdf

9. Organización de las Naciones Unidas (ONU). Objetivos y metas de desarrollo sostenible [Internet] [citado 2016 jul. 31]. Disponible en: https://www.un.org/sustainabledevelopment/es/objetivos-de-desarrollo-sostenible/

10. Pérez LC. La configuración jurídica de los ajustes razonables. Madrid: Ediciones Cinca; 2012 [citado 2016 sept. 12]. p. 159-83. Disponible en: https://www.convenciondiscapacidad.es/Noticias/la\%20configuracion\%20juri dica\%20de\%20los\%20ajustes\%20razonables.pdf

11. Organización de las Naciones Unidas (ONU). Convención sobre los derechos de las personas con discapacidad [Internet]. 2006 p. 35. Disponible en: https://www.joomla.corteidh.or.cr:8080/joomla/images/stories/Observ aciones/2/Anexo\%20XVII/L-8661\%20convenci\%C3\%B3n\%20d\%20pe\%20con\%20discapa.doc

12. Zondek A. Manual para una inclusión laboral efectiva de personas con discapacidad [Internet]. Chile: Servicio Nacional de Capacitación y Empleo; 2015 [citado 2016 jul. 25]. Disponible en: https://www.senadis.gob.cl/d escarga/i/2874/documento 
13. Nussbaum MCN. Las fronteras de la justicia. Consideraciones sobre la exclusión. 2012. Barcelona: Espasa Libros; 2012.

14. Sen A. La idea de la justicia. Bogotá: Taurus Pensamiento; 2011.

15. Nussbaum M, Sen A. La calidad de vida. México: The United Nations University; 2004.

16. Nussbaum M. Creating capabilities: the human development approach [Internet]. Cambridge, Mass: Belknap Press of Harvard University Press; 2011 [citado 2015 jun. 12]. Disponible en: https://www.cuadernosjudaicos.cl/in dex.php/ANUC/article/viewFile/31834/33629

17. Restrepo DA. ¿Qué entendemos por salud pública? En: Psicología, salud mental y salud pública. Medellín: Editorial CES; 2014.

18. Rodríguez V. Conociendo sobre revisiones sistemáticas. Rev Arch Col Med [Internet]. 2008 [citado 2018 mayo 20];I(2):64-8. Disponible en: https://colegiomedico.org.sv/conociendo-sobre-revisiones-sistematicas/

19. Finsterbusch C. La extensión de los ajustes razonables en el derecho de las personas en situación de discapacidad de acuerdo al enfoque social de derechos humanos. Rev Ius Prax [Internet]. 2016 [citado 2017 oct. 13];2:227-51. Disponible en: https://www.scielo.cl/pdf/iusetp/v22n2/art08.pdf

20. De Menezes JB, De Menezes HJB, De Menezes AB. A abordagem da deficiência em face da expansão dos direitos humanos. Rev Direitos E Garantias Fundam. 2016;17(2):551-72.

21. Ferrante C. Discapacidad y mendicidad en la era de la Convención: ¿postal del pasado? Converg Rev Cienc Soc [Internet]. 2015 [citado 2016 jul. 24 2016];68:151. Disponible en: https://www.redalyc.org/articulo.oa?id=1 0536227006

22. Victoria M. Hacia un modelo de atención a la discapacidad basado en los derechos humanos. Bol Mex Derecho Comp [Internet]. 2013 [citado 2013 dic. 1.o];46(138):1093-109. Disponible en: https://www.sciencedirect.co $\mathrm{m} /$ science/article/pii/S0041863313711621

23. De Asís R. Derechos humanos y discapacidad. Algunas reflexiones derivadas del análisis de la discapacidad desde la teoría de los derechos. En: Sobre discapacidad y derechos [Internet]. Madrid: Dykinson; 2007 [citado 2015 jun. 14]. p. 17-50. Disponible en: https://www.e-archivo.uc3m.es/handle/10016/9401

24. Palacios A. El modelo social de discapacidad: orígenes, caracterización y plasmación en la Convención Internacional sobre los Derechos de las Personas con Discapacidad [Internet]. Madrid: CERMI; 2008. Disponible en: https://www.cermi.es/es-ES/ColeccionesCermi/Cermi.es/Lists/Coleccion/Attachments/64/ Elmodelosocialdediscapacidad.pdf

25. Franco S. Los verbos esenciales de la salud pública. En: Granda Ugalde E. La salud y la vida [Internet]. Quito: Organización Panamericana de la Salud; 2011 [citado 2107 nov. 13. Disponible en: https://www.observatorio rh.org/sites/default/files/webfiles/fulltext/saludyvida_vol3.pdf

26. Cavalheiro G, Dornelles S, De Souza F, Itayra M, Cavalcanti L, Fernandes AM. Perfil das pessoas com deficiência física e Políticas Públicas: a distância entre intenções e gestos. Ciênc Saúde Coletiva [Internet]. 2016 [citado 2017 oct. 13];21(10):3131-42. Disponible en: https://www.scielo.br/scielo.php?script=sci_arttext\&pid=S1413-81 232016001003131\&lng=pt\&tlng=pt

27. Ramos S. La Convención sobre los derechos de las personas con discapacidad en salud mental. Una cuestión de derechos humanos. Rev Psiquiatr Salud Ment [Internet]. 2016 [citado 2016 jun 1.o];9(2):126-7. Disponible en: https://www.sciencedirect.com/science/article/pii/S188898911500138X

28. Sánchez P. Primera infancia y discapacidad en Chile. Revisión y enfoque actual a los programas de gobierno. Tema Cent Enfermedades Raras [Internet]. 2015;26(4):520-6. Disponible en: https://www.sciencedirect.com/scien ce/article/pii/S0716864015001017

29. Comisión Económica para América Latina y el Caribe (Cepal). El enfoque de derechos en la política laboral y salarial: construcción de un marco metodológico para aplicarse en Costa Rica, El Salvador, Guatemala, Nicaragua y México [Internet]. México: Naciones Unidas; 2013 [citado 2015 oct. 9]. Disponible en: https://www.reposit orio.cepal.org/bitstream/handle/11362/27176/LCMEXL1135s_es.pdf?sequence $=1$

30. Galvão LAC, Finkelman J, Henao S. Determinantes ambientales y sociales de la salud. Washington D. C.: Organización Panamericana de la Salud; 2010. 
31. Congreso de la República. Código Civil [Internet]. Ley 57 1887. Disponible en: https://www.alcaldiabogota.go v.co/sisjur/normas/Norma1.jsp?i=39535

32. Paolinelli C, González M. Epidemiología de la discapacidad en Chile, niños y adultos. Tema Cent Med Física Rehabil [Internet]. 2014;25(2):177-82. Disponible en: http://www.sciencedirect.com/science/article/pii/S07 16864014700288

33. Organización de las Naciones Unidas (ONU). Informe alternativo de la coalición colombiana para la implementación de la Convención sobre los derechos de las personas con discapacidad [Internet]. Ginebra: Organización de las Naciones Unidas; 2016 [citado 2016 sept. 22]. Disponible en: https://doc...

34. Ramos S. Igual reconocimiento de las personas con discapacidad ante la ley. Algunos comentarios sobre la "Convención sobre los derechos de las personas con discapacidad". FMC - Form Médica Contin En Aten Primaria [Internet]. 2016 [citado 2016 jul. 1.o];23(6):339-46. Disponible en: https://www.sciencedirect.com/science/a rticle/pii/S113420721630202X

35. Pino J, Valderrama C. Análisis desde el enfoque de derechos a la política chilena en discapacidad mental. Rev Chil Salud Pública. 2015;19(3).

36. Pelli L. A efetivação dos direitos das pessoas com deficiência pelos sistemas de proteção dos direitos humanos: sistema americano e europeu. Direitos Sociais E Políticas Públicas UNIFAFIBE [Internet]. 2012 [citado 2017 oct. 21];2. Disponible en: https://faef.revista.inf.br/imagens_arquivos/arquivos_destaque/XpIJi4SKLO7rVtt _2013-12-4-17-41-52.pdf

37. Rozo C. Principios bioéticos y discapacidad: la perspectiva de su inclusión en las políticas públicas. Rev Colomb Bioét. 2011;6(2):114-35.

38. Parra C, Palacios MT. Enfoque de derechos humanos en la política pública de discapacidad. Civilizar Cienc Soc Humanas [Internet]. 2007;7(13). Disponible en: https://www.redalyc.org/pdf/1002/100221524006.pdf

39. Reyes A. La proteccion de las personas con discapacidad en la Convención Americana sobre Derechos Humanos desde el enfoque de las capacidades: de la igualdad de trato a la igualdad de oportunidades. Am Univ Int Law Rev [Internet]. 2015 [citado 2017 mayo 4];30:249-84. Disponible en: https://www.litigation-essentials.lexisnexis.com/webcd/app?action=DocumentDisplay\&crawlid=1\&doct ype $=$ cite\&docid $=30+$ Am. + U. + Int\%27l+L. + Rev. $+249 \&$ srctype $=$ smi\&srcid $=3 B 15 \& k e y=c f d 0 f 5916 a 22 \mathrm{e} 1 \mathrm{~b} 5$ 89435 aed $408598 \mathrm{e} 0$

40. Hammell KW. Quality of life, participation and occupational rights: A capabilities perspective. Aust Occup Ther J [Internet]. 2015 [citado 2016 jul. 24];62(2):78-85. Disponible en: https://www.onlinelibrary.wiley.com/doi $/ 10.1111 / 1440-1630.12183 /$ abstract

41. Bellanca N, Biggeri M, Marchetta F. An extension of the capability approach: Towards a theory of dis-capability. ALTER - Eur J Disabil Res [Internet]. 2011 [citado 2017 nov. 7];5(3):158-76. Disponible en: https://www.lin kinghub.elsevier.com/retrieve/pii/S1875067211000514

42. Torres HF, Molina DC. Justicia, igualdad, discapacidad: una reflexión desde el "enfoque de las capacidades" y la teoría de la justicia de John Rawls. Rev Chil "Ter Ocupacional” [Internet]. 2014 [citado 2016 jul. 19];14(2):71-82. Disponible en: https://www.revistaestudiosarabes.uchile.cl/index.php/RTO/article/viewArt icle/35711

43. Di Tullio A. ¿ Hacia una justicia sin fronteras? El enfoque de las capacidades de Martha Nussbaum y los límites de la justicia. Daimon Rev Int Filos [Internet]. 2013 [citado 2017 mayo 4];(58):51-68. Disponible en: https://ww w.revistas.um.es/daimon/article/view/144611

44. Moreno M, Cortés E, Cárdenas A, Giraldo Z, Mena L. Valoración ocupacional de las personas con discapacidad, desde la perspectiva de las capacidades humanas. Rev Salud Pública [Internet]. 2013 [citado 2017 oct. 13];15(5). Disponible en: https://www.redalyc.org/html/422/42230941010/

45. Reicher SC. Human diversity and asymmetries: a reinterpretation of the social contract under the capabilities approach. SUR- Int J Hum Rights [Internet]. 2011 [citado 2017 oct. 13];14:167-79. Disponible en: https://w ww.heinonline.org/hol-cgi-bin/get_pdf.cgi?handle=hein.journals/surij14\$ion=12 
46. García N. El "enfoque de las capacidades" de Nussbaum y el concepto de "discapacidad”. Rev Estud Filos [Internet]. 2009 [citado 2017 mayo 4]; Disponible en: https://www.revistalegein.univalle.edu.co/documentos/legein9/G arciaNussbaum_n9.pdf

47. Toboso M, Arnau M. La discapacidad dentro del enfoque de capacidades y funcionamientos de Amartya Sen. Rev Araucaria [Internet]. 2008 [citado 2015 abr. 12];20:1-39. Disponible en: https://www.digital.csic.es/handle/1 $0261 / 23277$

48. Mitra S. The capability approach and disability. J Disabil Policy Stud [Internet]. 2006 [citado 2016 sept. 11];16(4):236-47. Disponible en: https://www.dps.sagepub.com/content/16/4/236.short

49. Burchardt T. Capabilities and disability: the capabilities framework and the social model of disability. Disability and Society [Internet]. 2004 [citado 2016 sept. 11];19(7):735-51. Disponible en: https://www.staging.scie-socialcareonline.org.uk/capabilities-and-disability-the-capabilities-framework-an d-the-social-model-of-disability/r/a1CG0000000GSyQMAW

50. Strapazzon C, Pinheiro M. Direitos humanos fundamentais das pessoas com deficiências: the capability approach. Rev AJURIS [Internet]. 2014 [citado 2017 oct. 14];41(133):155-83. Disponible en: https://www.ajuris.org.br /OJS2/index.php/REVAJURIS/article/view/224

51. Zetina E. Necesidades capacidades y derechos humanos bajo la perspectiva de Martha Nussbaum [Internet]. México: Universidad Autónoma del Estado de México; 2016 [citado 2017 mar. 30]. Disponible en: https://www.ri.uaemex.mx/bitstream/handle/20.500.11799/65250/Necesidades\%20capacidades\%20y\%2 0derechos\%20humanos\%20bajo\%20la\%20perspecti.pdf?sequence $=1$

52. Urmeneta X. Discapacidad y derechos humanos. Norte Salud Ment [Internet]. 2010 [citado 2017 mayo 4];8(38):65-74. Disponible en: https://www.dialnet.unirioja.es/descarga/articulo/4830148.pdf

53. Nussbaum MC. Capabilities and human rights. Fordham Rev [Internet]. 1997 [citado 2016 sept. 11];66:273-300. Disponible en: https://www.heinonline.org/hol-cgi-bin/get_pdf.cgi?handle=hein.journals/flr66@ion=18

54. Calderón G. Bioética, derechos y capacidades humanas. Bogotá: Pontificia Universidad Javeriana; 2012.

55. Reyes CMR, Robayo AMM. Discapacidad y justicia distributiva: una mirada desde la bioética. Rev Colomb Psiquiatr [Internet]. 2011;40(2):336-51. Disponible en: https://www.sciencedirect.com/science/article/pii/S0 $03474501460127 \mathrm{X}$

\section{Licencia Creative Commons CC BY 4.0}

Para citar este artículo: Gómez Rúa NE, Restrepo Ochoa DA, Gañan Echavarría J, Cardona Arango D. La discapacidad desde los enfoques de derechos y capacidades y su relación con la salud pública. Rev. Gerenc. Polit. Salud. 2018;17(35). https://www.doi.org/10.11144/Javeriana.rgps17-35.dedc 